\title{
3 Homecare und Entlassmanagement
}

Die Homecare-Versorgung setzt oft an, wenn ein Patient oder eine Patientin das Krankenhaus verlässt und in die eigene Häuslichkeit oder das betreute Wohnumfeld zurückkommt, wo die Versorgung fortgeführt werden muss. Dem vorgelagert oder parallel läuft ein wichtiger Prozess der Versorgung: der Übergang aus einem Versorgungssektor in einen anderen. Der Übertritt aus dem stationären in den ambulanten, rehabilitativen oder pflegerischen Sektor (letztere auch stationär) stellt in der deutschen (oder jeglichen) Versorgungslandschaft eine Schnittstelle dar, die oftmals nicht ausreichend und selten optimal bedient ist (Deimel et al., 2012).

So benötigen Patienten bei und unmittelbar nach der Entlassung meist eine intensive medizinische Zuwendung, die auf die Therapie der Klinik abgestimmt ist. Damit jedoch ist der zuständige Hausarzt häufig unzureichend vertraut (Harbord, 2009). Daneben kann es vor allem bei Entlassungen, die an einem Freitag erfolgen, zu Versorgungsbrüchen auf Grund der mangelnden Erreichbarkeit der nachsorgenden Ärzte oder weiteren Leistungserbringer kommen. Aus diesem Crund wird ein professionelles Entlassmanagement eingesetzt (Harbord, 2009). Der Begriff umfasst dabei jegliche Form der Überleitung aus dem Krankenhaus in die ambulante oder stationäre Nachsorge (DKI, 2014).

Ziel ist es, durch systematisches Management des Entlass- bzw. Übergangsprozesses die Versorgungskontinuität zu gewährleisten. Denn: Die Überlei- 
tung erfordert einen hohen koordinativen Aufwand. Um dies zu erreichen, müssen die Akteure unterschiedlichster Versorgungsbereiche und Sektoren kommunizieren und Informationen austauschen (Mohr, 2009). Gleichzeitig müssen medizinische sowie finanzielle Verantwortlichkeiten geklärt werden. Daher ist es nicht nur wichtig, diesen Prozess gut zu planen, sondern auch rechtlich abzusichern, um eine nahtlose Versorgung der Patienten gewährleisten zu können. Die Rolle von Homecare-Fachkräften für das nahtlose Abwickeln dieses Prozesses wird vermehrt hervorgehoben, da diese oftmals Kommunikations- und Koordinationsaufgaben übernehmen (BVMed, 2014; Harbord, 2009).

Das folgende Kapitel geht zunächst auf die Notwendigkeit eines Entlassmanagements sowie die Komplexität des Prozesses ein. Anschließend werden bestehende Hürden und Barrieren sowie die rechtlichen Regelungen in Deutschland dargestellt. Ein kurzer Exkurs in die Rolle von Homecare-Unternehmen im Entlassmanagement beendet dieses Kapitel.

\subsection{Eine (neue) Notwendigkeit}

Der Bedarf für ein angemessenes Entlassmanagement hat sich in den letzten Jahrzehnten gewandelt. Ein zentraler Aspekt, der in diesem Zusammenhang oftmals genannt wird, ist die Einführung des DRG-Entgeltsystems im stationären Sektor. In den 197oer-Jahren war es noch üblich, bei der Entlassung der Patienten Aspekte wie die individuelle Lebenssituation einzubeziehen (zum Beispiel: Lebt der Patient allein? Gibt es Angehörige, Freunde oder Nachbarn, die die Versorgung unterstützen können?). Die Entlassung wurde teilweise sogar so lang hinausgezögert, bis der Patient für sich sorgen konnte. Solch ein Vorgehen ist unter dem heutigen Abrechnungssystem in den Krankenhäusern (zumindest in dem Maße) nicht mehr durchführbar (Mohr, 2009). Die durchschnittliche Verweildauer im stationären Sektor hat sich in den letzten 40 bis 50 Jahren in Deutschland deutlich reduziert, obwohl sie im europäischen Vergleich noch immer hoch ist (Mohr, 2009).

Das heißt zwar nicht zwangsläufig, dass die Patienten heute zu früh entlassen werden, es bedeutet aber, dass die verfügbare Zeit, den sozialen und auf das Lebensumfeld bezogenen Kontext der Patienten miteinzubeziehen, gesunken ist. Die Entlassung der Patienten erfolgt nicht mehr unter Berücksichtigung der oben genannten Faktoren. Vielmehr befinden sich die Krankenhäuser heutzutage „in einem entscheidenden Veränderungs- und Konzentrationsprozess", der unter anderem mit Kostendämpfungen einhergeht, wodurch der Entlassungsprozess neuen Rahmenbedingungen ausgesetzt ist (Mohr, 2009).

Häufig wird in diesem Zusammenhang der „Drehtüreffekt“ erwähnt: Patienten werden „zu früh“ entlassen, um anschließend erneut eingewiesen 
zu werden - auch, weil sie nicht in der Lage sind, die Versorgung im ambulanten Bereich weiterzuverfolgen. Zahlen aus den USA zeigen dies deutlich: Etwa ein Fünftel der Medicare-Bezieher, die aus dem Krankenhaus entlassen werden, wird innerhalb von 30 Tagen wieder hospitalisiert. Die Hälfte dieser Patienten hatte in der Zeit zwischen diesen beiden Episoden keinen Kontakt mit einem Hausarzt (Hennessey \& Suter, 2011; Rennke et al., 2013).

Doch nicht nur neue Abrechnungsmodalitäten und Finanzierungsmodelle, auch gesellschaftliche Aspekte und medizinischer Fortschritt führen dazu, dass Entlassmanagement an Bedeutung gewinnt. Viele der Studien und Berichte, die das Thema Entlassmanagement beleuchten, führen in die Thematik ein, indem sie auf die Notwendigkeit, Kosten einzusparen, verweisen. Im zweiten Atemzug werden Aspekte wie Empowerment, Patientenzufriedenheit oder medizinische Outcomes aber auch demografische Faktoren genannt.

Demografisch erleben wir nicht nur den Trend, dass Menschen heutzutage immer länger leben - sie tun dies auch vermehrt in Einzelhaushalten, ohne die Unterstützung von Familie oder Nachbarschaften. Chronische Erkrankungen treten im Alter nicht nur vermehrt, sondern oft auch gleichzeitig mit weiteren (chronischen) Erkrankungen auf. Dies alles führt dazu, dass es heute in unserer Gesellschaft immer mehr Menschen gibt, die lange und wiederholt auf pflegerische oder medizinische Hilfe und Versorgung angewiesen sind. So macht die ältere Bevölkerung in den USA und Kanada nur 13\% bzw. 14\% der Bevölkerung aus, jedoch entfallen 45\% bzw. 50\% der Krankenhausausgaben auf diese Gruppe (Fox et al., 2013).

Es kommt hinzu, dass heutzutage viele Eingriffe, die ehemals stationär durchgeführt werden mussten, ambulant erfolgen. Dies bedeutet, dass sich das Profil der Patienten, die für Eingriffe den stationären Sektor aufsuchen, verändert. Jene Patienten, die für Operationen ins Krankenhaus gehen, sind oftmals dadurch charakterisiert, dass sie einen besonderen Versorgungsbedarf aufweisen. Stationär behandelte Patienten sind oftmals älter, leiden unter vielen Erkrankungen (Multimorbidität) und sind besonders bedürftig (Abad-Corpa et al., 2013; Mohr, 2009).

Mit der erwähnten verkürzten Zeit im stationären Aufenthalt, aber auch den weiteren sozialpolitischen und gesellschaftlichen Veränderungen, bekommt der erfolgreiche Übergang vom stationären in den ambulanten Sektor besondere Relevanz. Es gilt, die finanziellen, sozialen, demografischen und medizinischen Faktoren so einzuarbeiten, dass der Übergangsprozess diesen gerecht wird und eine qualitativ hochwertige Versorgung weiterhin gewährleistet werden kann.

Schätzungen zufolge wurden in den USA Ende der 8oer-Jahre etwa 30\% der Entlassungen aus dem Krankenhaus aus nicht-medizinischen Gründen verzögert. Ein ähnlicher Zustand wurde auch noch für das Jahr 2003 ange- 
nommen. Zu diesen nicht-medizinischen Gründen zählten folgende: Nicht adäquates Assessment der Patienten durch Gesundheitspersonal und damit einhergehend nicht ausreichendes Wissen über das soziale Umfeld eines Patienten; Probleme bei der Organisation der Versorgung nach der Entlassung, zu spätes Buchen von Transportmöglichkeiten der Patienten, und mangelhafte Kommunikation zwischen Krankenhaus, Pflege und Leistungserbringern im ambulanten Bereich (Shepperd et al., 2013).

Vermeidbare Wiedereinweisungen aber auch unnötig lange verzögerte Entlassungen verweisen auf fehlendes oder unzureichendes Entlassmanagement. Patienten werden allein gelassen, obwohl sie ihre Funktionsfähigkeit noch nicht ausreichend zurückerlangt haben. Vor allem bei multimorbiden Patienten ist oftmals unklar, welche Ärzte in der Phase nach der Entlassung die Betreuung übernehmen (Hennessey \& Suter, 2011). Aber auch Aspekte wie die kontinuierliche Versorgung der Patienten mit Arznei- sowie Hilfs- und Heilmitteln sind von entscheidender Bedeutung für den Behandlungserfolg.

Vor diesem Kontext ist das Ziel des Entlassmanagements, die Effizienz aber auch die Qualität der Gesundheitsversorgung unter Berücksichtigung all dieser Faktoren zu verbessern. Wong et al. (2011, S. 2) sehen vor allem zwei Aufgaben beim Entlassmanagement:

„Today, there are mainly two different purposes underpinning discharge planning. One is to transfer both care and budgetary responsibility from the hospital to other agencies for patients who are not in need of in-patient care. Another purpose is to plan for patients' continuing health and social care."

Dabei muss klar sein: Erfolgreiches Entlassmanagement gibt es nicht umsonst. Die Organisation eines erfolgreichen Entlassprozesses kann und wird Investitionen benötigen und zunächst Kosten verursachen (Shepperd et al., 2013). Insgesamt jedoch birgt es großes Potential für die Wirtschaftlichkeit der Gesundheitssysteme. Es konnte bereits gezeigt werden, dass Entlassmanagement zu Einsparungen in der Gesundheitsversorgung führen kann (Shepperd et al., 2013). Ressourcen können effizienter eingesetzt, bessere medizinische Ergebnisse erzielt sowie durch Wiedereinweisungen verursachte Kosten teilweise vermieden werden (Abad-Corpa et al., 2013, vgl. Abbildung 15).

\subsubsection{Entlassmanagement heißt Management von Komplexität}

„Effective discharge planning requires capacity planning, performance review, hospital discharge policies, and healthcare providers/stakeholders agreements. There is clear evidence and wide agreement among healthcare providers/stakeholders that a standardized and policy-driven protocol [is] important to an effective discharge planning." (Wong et al., 2011, S. 9) 


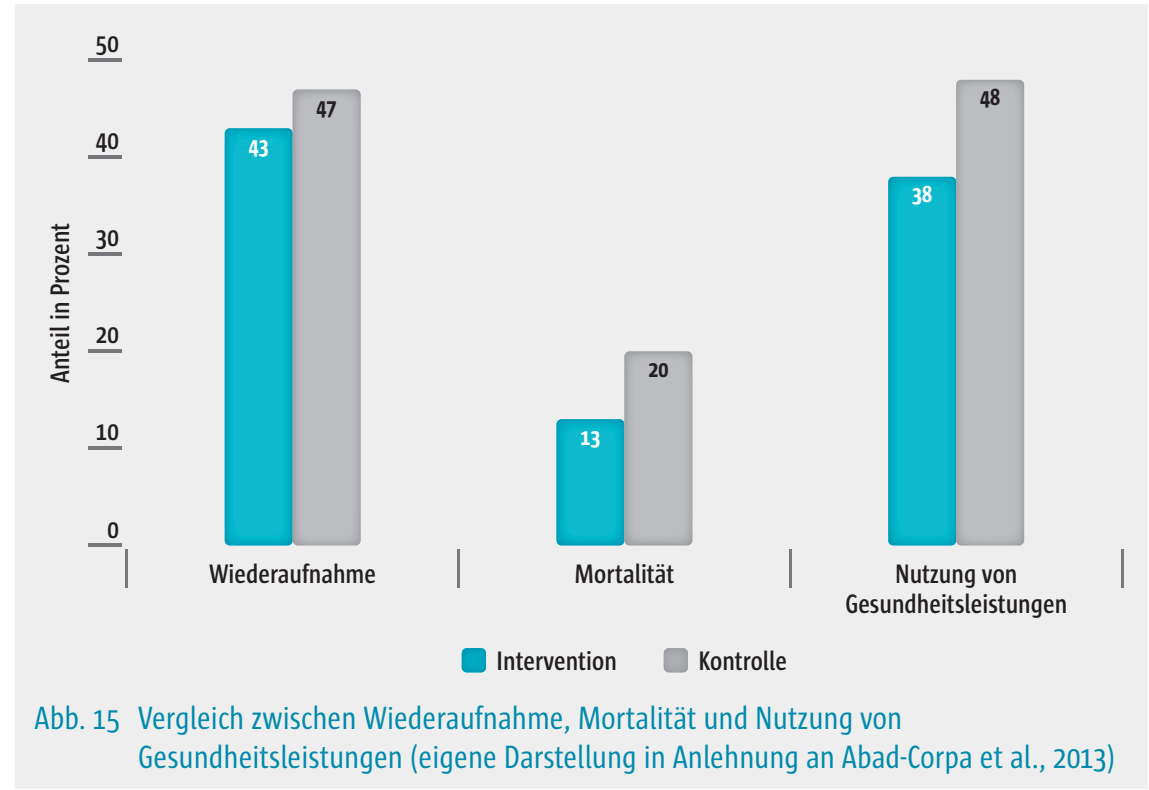

Der Prozess der Entlassung eines Patienten aus dem stationären Sektor des Krankenhauses in den ambulanten oder den stationären pflegerischen Sektor ist allein auf Grund der verschiedenen Bereiche, die in diesem Prozess involviert sind, komplex. Laut Deimel et al. (2012) gibt es sechs Bereiche, die nach der Entlassung einbezogen werden können: Medizin, Rehabilitation, Pflege, Hilfsmittel, Sozial, Angehörige (vgl. Tabelle 2). Meistens (wenn nicht immer) ist es jedoch nicht exklusiv einer dieser Bereiche, der mit dem Krankenhaus eine Schnittstelle bildet - vielmehr ist davon auszugehen, dass ein Großteil der Bereiche gleichzeitig relevant ist.

Deutlich wird, dass nicht nur professionelle Versorger, sondern auch das soziale Umfeld und soziale Dienste zentral sind. Das Entlassmanagement ist somit ein multidisziplinäres Unterfangen, welches nicht nur viele Akteure, sondern auch Lebensbereiche des Patienten umfasst.

Entlassmanagement ist ein umso komplexeres Unterfangen, da an dieser Schnittstelle nicht nur unterschiedliche Akteure zusammenkommen. Es treffen auch unterschiedliche Abrechnungssysteme und Versorgungssektoren aufeinander. Vor allem die Tatsache, dass unterschiedliche Kostenträger in einem Prozess zusammengebracht werden müssen, erschwert die Aufgabe, dem Patienten eine kontinuierliche qualitativ hochwertige Versorgung zu garantieren. Tabelle 3 stellt dar, welche Kostenträger für die einzelnen Nachsorgebereiche verantwortlich sind.

Dass argumentiert wird, es bedürfe eines qualifizierten Entlassmanagers, einer verantwortlichen Person, die sich der Prozesse annimmt, ist daher 
Tab. 2 An der Nachsorge beteiligte Bereiche im Entlassmanagement (eigene Darstellung in Anlehnung an Deimel et al., 2012)

\title{
Bereiche der Nachsorge
}

\section{Medizin}

- Haus- und fachärztliche Betreuung

- Diagnostik

- Therapie in einem anderen Krankenhaus

- Wundmanagement

- Ernährungstherapie

- Arzneimittelmanagement

\section{Rehabilitation}

- ambulante und stationäre Rehabilitation

- Physiotherapie

- Ergotherapie

- Logopädie

\section{Sozial}

- Wohnungs- und Finanzierungsfragen

- psychosozialer Dienst

- Schwerbehinderung

\section{Pflege}

- ambulante Pflegedienste

- Pflegeberatung

n teilstationäre/stationäre Pflegeeinrichtungen
Hilfsmittel
- Fortbewegung
- häusliche Unterstützung
- ggf. Medizinprodukte

\author{
Angehörige \\ - ambulante Hilfsangebote \\ - stationäre Pflegeeinrichtungen \\ - Kurzzeitpflege
}

Tab. 3 Nachsorgebereiche und verantwortliche Kostenträger (eigene Darstellung in Anlehnung an Deimel et al., 2012)

\begin{tabular}{lcccc} 
Nachsorgebereich & $\begin{array}{c}\text { KrankenV } \\
\text { SGB V }\end{array}$ & $\begin{array}{c}\text { PflegeV } \\
\text { SGB XI }\end{array}$ & $\begin{array}{c}\text { BG Kostenträger } \\
\text { SGB VIII }\end{array}$ & Sozialamt \\
\hline Medizin & + & & + & \\
\hline Rehabilitation & + & + & + & + \\
\hline Pflege & ++ & + & + & + \\
\hline Hilfsmittel & + & + & + & + \\
\hline Sozial & & & + & \\
\hline Angehörige & & + & & \\
\hline
\end{tabular}

kaum verwunderlich (Deimel et al., 2012; Harbord, 2009; Hennessey \& Suter, 2011; Wong et al., 2011). Die Definition einer klar zugeordneten und verantwortlichen Person hat sich vor allem für das Aufgabenfeld der Kommunikationsübernahme bewährt (Wong et al., 2011). Denn um Entlassmanagement erfolgreich und effektiv zu betreiben, bedarf es der Kommunikation zwischen Akteuren, die im Versorgungsalltag ansonsten oftmals wenig bis gar nicht miteinander arbeiten (Mohr, 2009). Aus Sicht der nachsorgenden Ärzte ist sicherlich der Entlassbrief ein zentrales Element der Kommunikation zwischen den Versorgern. Dieser sollte Diagnose, Therapievorschläge inklu- 
sive der Medikation, Angaben zur Heil- und Hilfsmittelversorgung sowie zur häuslichen Krankenpflege aufführen. Auch Informationen zu Kontrollterminen sollten darin enthalten sein (Reus, 2012).

Neben einer klar zugeordneten Rollen- und Verantwortlichkeitsdefinition sowie der Kommunikation ist frühzeitiges Initiieren des Entlassmanagements zentral, um all die unterschiedlichen Bereiche rechtzeitig ansprechen und koordinieren zu können (Harbord, 2009). Dies sollte auch das Screening von Hochrisikopatienten beinhalten, um Drehtüreffekte zu vermeiden (Wong et al., 2011).

In dem Review von Fox et al. (2013) wurde die Effektivität eines solchen frühzeitig initiierten Entlassmanagements im Vergleich zur Regelversorgung untersucht. Betrachtet wurden Faktoren wie Krankenhausaufenthaltsdauer, Wiedereinweisungsrate und Dauer der Wiedereinweisung, Sterberate, Zufriedenheit und Lebensqualität der Patienten. Frühzeitiges Entlassmanagement wurde dadurch definiert, dass das Management bereits in der akuten Phase der Erkrankung initiiert und sobald die akute Phase überstanden ist, eingeleitet wurde. Die Meta-Analyse zeigte, dass die Krankenhausaufenthaltsdauer zwar nicht signifikant kürzer, die Wiedereinweisungsrate jedoch nach einem bzw. zwölf Monaten signifikant geringer war. Und auch die Dauer des Aufenthalts bei Wiedereinweisung konnte durch frühzeitiges Entlassmanagement im Schnitt um fast zweieinhalb Tage verkürzt werden. Die Mortalität unterschied sich nicht signifikant zwischen den beiden betrachteten Gruppen in den Studien.

Doch nicht nur frühzeitig initiiertes Entlassmanagement, sondern jegliches Entlassmanagement hat positive Effekte. Zwei weitere Reviews zeigten, dass es zum einen zu einer verringerten Krankenhausaufenthaltsdauer (vor allem bei älteren Patienten) führen kann (Shepperd et al., 2013). Bestätigt wurde zudem, dass die Wiedereinweisungsraten durch das Entlassmanagement gesenkt werden (Rennke et al., 2013; Shepperd et al., 2013).

Studien zeigen zudem, dass Patienten, die ein Entlassmanagement erhielten, mit der Versorgung im Krankenhaus und während der Entlassung zufriedener waren (Abad-Corpa et al., 2013; Shepperd et al., 2013). Die Ergebnisse zur gestiegenen Zufriedenheit der Patienten mit der Versorgung verweisen deutlich darauf, dass das Entlassmanagement einen Akteur beinhaltet, der bislang (zu) häufig ignoriert wird: den Patienten. Die bisher oftmals passive Rolle der Patienten muss und wird im Sinne eines erfolgreichen Übergangsprozesses geändert werden. Zudem zeigen Patientenbefragungen, dass Patienten sich eine stärkere Einbindung und Aufklärung wünschen (Mohr, 2009). Entlassmanagement soll daher nicht nur die Koordination verbessern, sondern auch den Patienten das Gefühl vermitteln, dass sie in den Entscheidungsprozess einbezogen sind (Abad-Corpa et al., 2013).

Laut Mohr (2009) war das Entlassmanagement bisher dominiert vom klassischen Arzt-Patientenverhältnis, in dem der Patient eine passive Rolle ein- 
genommen hat. Erkennbar war eine geringe Orientierung an dem Interesse des Patienten, die sich heute auch oft noch zeigt. Erfolgreiches Entlassmanagement benötigt jedoch eine Einbindung der Patienten in den Prozess als aktive Partner mit individuellen Wünschen und Vorlieben. Insbesondere die Idee des selbstbestimmten Patienten, der die eigene Gesundheit selbst in der Hand hat und diese verantwortet, verlagert (Mit-)Verantwortung für die eigene Gesundheit. Vom Patienten wird nunmehr erwartet, die Rolle des Experten in eigener Sache anzunehmen. Dies muss jedoch auch von den Leistungserbringern des Systems gefördert und respektiert werden. Die notwendigen Komponenten dafür sind insbesondere Information und Aufklärung (Mohr, 2009).

\subsubsection{Hürden und Barrieren für das Entlassmanagement}

Folgt man der Stellungnahme des Bundesverbands Managed Care (Deimel et al., 2012), dann gibt es folgende Gründe dafür, dass das Entlassmanagement bislang nicht ausreichend etabliert und geregelt ist:

- Die Anreize zur Durchführung und Etablierung langfristiger und spezialisierter Strukturen sind zu gering. Dadurch bedingt gibt es starke Unterschiede in der Qualität des Entlassmanagements in den Krankenhäusern.

- Es bestehen zu viele bürokratische Hürden. Als Beispiele werden etwa das aufwändige Einholen von Kostenzusagen und der Genehmigungsvorbehalt der Kassen im Bereich der Rehabilitation aufgeführt.

- Patienten als auch Angehörige sind unzureichend informiert.

- Die Kontinuität der Versorgung wird durch fehlerhafte oder unvollständige Informationsweitergabe gefährdet.

- Es fehlen die Ansprechpartner für die pflegerische sowie soziale Weiterbetreuung im ambulanten Bereich.

Diese aufgeführten Hürden speisen sich deutlich aus der bereits dargestellten Komplexität des Prozesses. Dass diese Komplexität kein spezifisch deutsches Phänomen ist, zeigt sich an den Ergebnissen einer Studie von Wong et al. (2011, vgl. Abbildung 16). Sie untersuchte die aktuelle Praxis des Entlassmanagements sowie Barrieren für eine erfolgreiche Durchführung und Etablierung in Hongkong und fand vier Problemfelder.

Die Systemebene birgt Hürden durch mangelnde Infrastruktur, unzureichende rechtliche Regelungen, fehlende Richtlinien sowie fehlende Flexibilität der Regelversorgung. Die Kommunikation zwischen den Akteuren wird unnötigerweise erschwert und die Informationsweitergabe ist problematisch. Oftmals werden Patienten frühzeitig entlassen, ohne dass das System in der Lage ist, direkt erfolgreiche Anschlussbehandlungen zu garantieren. Die Leistungserbringer stellen eine Hürde dar, wenn zum Beispiel Ärzte und 




Abb. 16 Spannungsfeld der Hürden und Barrieren für erfolgreiches Entlassmanagement (eigene Darstellung in Anlehnung an Wong et al., 2011)

Krankenschwestern sowie Pfleger nicht ausreichend über die Bedürfnisse der Patienten informiert sind oder diese Akteure die Behandlung der Patienten nicht eindeutig und ausreichend dokumentieren. Auch hier ist ein Informationsdefizit bzw. die fehlende Weitergabe der Informationen zentral. Patienten sind oftmals unzureichend aufgeklärt und können ihre Präferenzen für den Entlassungsprozess nicht formulieren. Die sozialen Aspekte, die als Hürden wahrgenommen werden, umfassen u.a. mangelnde Kommunikationsfähigkeit zwischen den Disziplinen, mangelnde Infrastruktur (z.B. mit Blick auf den Transport des Patienten aus dem Krankenhaus in die Häuslichkeit oder Ähnliches), lange Wartezeiten auf den ersten Kontakt nach Entlassung als auch die Herausforderungen bedingt durch die sozio-ökonomische Situation des Patienten (u.a. Zahlungsfähigkeit) (Wong et al., 2011).

Ähnlich sind auch die Gründe, die für das Hinauszögern der Entlassung von Patienten in den USA, sowie die vermeidbaren Einweisungen bedingt durch ungenügendes Entlassmanagement bereits weiter oben genannt wurden (Hennessey \& Suter, 2011; Rennke et al., 2013; Shepperd et al., 2013):

- mangelhaftes Assessment,

- unzureichende Kenntnis des sozio-ökonomischen Status der Patienten,

- Organisations-, Koordinations- und Abstimmungsprobleme sowie

- generell mangelhafte Kommunikation.

\subsubsection{Entlassmanagement in Deutschland}

International ist die Relevanz von Entlassmanagement vielerorts erkannt. Wie jedoch Entlassmanagement organisiert und reguliert wird unterschei- 
det sich zum Teil stark. Im Vereinigten Königreich formulieren die NHS Richtlinien seit 2004, dass jeder Patient bereits bei Einweisung ins Krankenhaus einen Entlassungsplan erhalten sollte. In den USA sind die Krankenhäuser legal zum Entlassmanagement verpflichtet, während in Hong Kong das Entlassmanagement und die Entlassplanung vom Hausarzt initiiert wird und es keine bindenden Regelungen dazu gibt (Wong et al., 2011). Die legale Verpflichtung schlägt sich u.a. darin nieder, dass das Center for Medicare \& Medicaid Services finanzielle Strafen in Höhe von bis zu 1\% der MedicareEinnahmen an Krankenhäuser verhängen kann, falls die Krankenhäuser zu hohe Wiedereinweisungsraten aufweisen (Rennke et al., 2013). Dennoch gebe es laut Rennke et al. (2013) bislang wenige effektive Entlassmanagement-Strategien. Vor allem die Vielzahl der unterschiedlichen Strategien und Herangehensweisen macht den Vergleich und die Synthese oder gar die Formulierung eines Goldstandards schwer (Rennke et al., 2013).

Im Review von Shepperd et al. (2013) zur Effektivität von Entlassmanagement zeigt sich, wie unterschiedlich es ausgestaltet sein kann. So kann das Entlassmanagement bereits vor oder bei Einweisung ins Krankenhaus oder aber erst drei Tage vor Entlassung angefangen werden. Zudem unterscheiden sich die Bereiche, die mit einbezogen werden (werden z.B. pharmakologische Aspekte ebenso bedacht wie soziale?) als auch die verantwortlichen Personen. Darauf wurde bereits im vorherigen Teil zur Komplexität eingegangen.

\section{Rechtliche Rahmenbedingungen des Entlassmanagements in Deutschland}

In der Organisation der Entlassung ist es essentiell, dass Kompetenzen und Zuständigkeiten klar definiert sind. In Deutschland hat es seit den 196oerJahren einen Wandel in der Zuständigkeit gegeben. Waren früher vor allem die Gemeindeschwestern für die Betreuung entlassener Patienten zuständig, wurde die Zuständigkeit in den 1980er-Jahren von unterschiedlichsten Dienstleistern, zu denen auch Sozialstationen, ambulante (zunächst freigemeinnützig tätige und seit den 199oer-Jahren vermehrt auch private) Pflegedienste getragen (Mohr, 2009). Heute liegt die Organisation des Entlassungsprozesses beim Krankenhaus und den dortigen Ärzten, unter Mitarbeit der Sozialdienste (Mohr, 2009).

Der Anspruch der Patienten auf ein Versorgungsmanagement, welches die Kontinuität der Behandlung und Betreuung garantiert, wurde im GKV-Wettbewerbsstärkungsgesetz (GKV-WSG) zum 01.04.2007 verankert. Nach $\mathbb{1} 11$ Abs. 4 SGB V wird der „Anspruch auf ein Versorgungsmanagement insbesondere zur Lösung von Problemen beim Übergang in die verschiedenen Versorgungsbereiche“ definiert. Dazu gehört auch der Übergang, der mit der Entlassung aus dem Krankenhaus erfolgt. Das Entlassmanagement wurde mit dem GKV-Versorgungsstrukturgesetz als Teil der Krankenhausbehandlung festgelegt. Laut $\$ 39$ Abs. 1a SGB V gilt: 
„Die Krankenhausbehandlung umfasst ein Entlassmanagement zur Unterstützung einer sektorenübergreifenden Versorgung der Versicherten beim Übergang in die Versorgung nach Krankenhausbehandlung.“

Mit dem GKV-Versorgungsstärkungsgesetz (GKV-VSG) werden die Krankenkassen weiter in das Entlassmanagement eingebunden. Sie sind mit dem Krankenhaus in der Pflicht, die Leistungserbringer frühzeitig zu kontaktieren und so dem unmittelbaren Rechtsanspruch auf ergänzende Unterstützung des Entlassmanagements, den die Versicherten gegenüber den Krankenkassen nun erhalten haben, auszufüllen.

Um die Patienten in dem Übergangszeitraum zu versorgen werden den Krankenhäusern mit dem GKV-VSG weitreichendere Rechte, was die Verschreibung von Arznei-, Verbands- und Hilfs/Heilmitteln angeht, zugeschrieben. Bislang waren die Krankenhäuser auf Rezeptverschreibungen der niedergelassenen Ärzte angewiesen (BVMed, 2014). Dies erschwerte die Organisation und Durchführung des Entlassmanagements. Mit Blick auf die Arzneimittelverschreibung wird festgelegt, dass die kleinste Packung gemäß Packungsgrößenverordnung verordnet werden darf. Daneben darf auch häusliche Krankenpflege sowie Soziotherapie verordnet und Arbeitsunfähigkeit festgestellt werden. Für einen Zeitraum von maximal sieben Tagen nach der Entlassung dürfen die Krankenhäuser ambulante Leistungen verordnen und werden somit für diesen Zeitraum den Vertragsärzten für maximal diesen Zeitraum gleichgestellt. Dennoch liegt die Verantwortung für die ambulante Versorgung nach wie vor bei den Vertragsärzten. Bislang richtet sich der Anspruch der Versicherten auf Entlassmanagement gegen die Krankenhäuser. Dies bleibt auch weiterhin bestehen. Jedoch räumt das GKV-VSG nun die Möglichkeit ein, dass die Verantwortung auch auf andere Leistungserbringer übertragen werden kann. Konkret steht nun in $\$ 39$ Abs. 1a:

„Das Krankenhaus kann mit Leistungserbringern nach \$95 Absatz 1 Satz 1 vereinbaren, dass diese Aufgaben des Entlassmanagements wahrnehmen."

Ein Rahmenvertrag zwischen dem Spitzenverband der Krankenkassen, der Kassenärztlichen Bundesvereinigung und der Deutschen Krankenhausgesellschaft sollte bis zum 31.12.2015 die Einzelheiten der Zusammenarbeit der Leistungserbringer mit den Krankenkassen regeln. Da man sich nicht einigen konnte, wurde der Rahmenvertrag im Schiedsverfahren vereinbart und tritt zum 01.07.2017 in Kraft.

Das Entlassmanagement sowie die Übertragung der dafür notwendigen Daten darf nur unter Einwilligung des Versicherten erfolgen. Dazu muss der Patient vorher informiert werden und dem schriftlich zustimmen. Daher ist es in der Praxis des Entlassmanagements zentral, dass die Einverständniserklärung der Patienten vorliegt (Reus, 2012). Erst dann können Prozesse initiiert werden. 


\section{Organisatorische Umsetzung des Entlassmanagements in deutschen Krankenhäusern}

Im Zeitraum Mai 2013 bis Juni 2014 führte das Deutsche Krankenhaus Institut (DKI) im Auftrag der Deutschen Krankenhausgesellschaft (DKC) eine Studie zum Entlassmanagement im Krankenhaus durch. Sie basiert auf einer Vollerhebung der Allgemeinkrankenhäuser ab 50 Betten mit einer Rücklaufquote von 43\% (673 Krankenhäuser). Die Ergebnisse zeigen, dass in drei Viertel der Krankenhäuser schriftliche Standards zum Entlassmanagement vorhanden sind. Dabei wurde vor allem der Nationale Expertenstandard „Entlassmanagement in der Pflege (DNQP)“ verwendet. Dieser wurde als ein Weg zur einheitlichen und strukturierten Lösung des Entlassmanagements eingeführt (Deimel et al., 2012). Außerdem hatten mehr als die Hälfte der Krankenhäuser (zusätzlich) hauseigene Standards definiert.

Es scheint, als würde die Notwendigkeit eines qualifizierten Personals erkannt: Zwei Drittel der Krankenhäuser verfügten über speziell für das Entlassmanagement zuständige Organisationseinheiten und in $80 \%$ der Krankenhäuser wurde speziell qualifiziertes Fachpersonal eingesetzt, um das Entlassmanagement durchzuführen.

Laut der Studie gibt es in Deutschland viele regionale Initiativen zum Entlassmanagement, an denen sich gut 88\% der Krankenhäuser bereits beteiligten und weitere $4 \%$ eine Beteiligung planen (DKI, 2014). Zu solchen Initiativen mag beispielhaft das Modellprojekt „Sicher nach Hause“ (SINAH) in SüdHessen zählen. In diesem werden jene Patienten angesprochen, die aus dem stationären Bereich in die eigene Häuslichkeit entlassen werden und noch auf Hilfe für die Bewältigung des Alltags angewiesen sind. In diesem Modellprojekt sind die SINAH-Begleiter/innen zentral, die den Patienten über einen Zeitraum von sechs bis acht Wochen nach der Entlassung besuchen und bei Besorgungen, Formalitäten oder Antragsstellungen unterstützen. Die SINAH kommen auf Anfrage des Sozialdienstes der Krankenhäuser zum Einsatz. Die Hausärzte, die ebenfalls integriert sind, können den Patienten bereits vor dem Krankenhausaufenthalt informieren und somit frühzeitig den Entlassungsprozess vorbereiten (Bördlein, 2015).

Mit Blick auf die Weiterentwicklung und Verbesserung des Entlassmanagements ist festzuhalten, dass lediglich 50\% der Krankenhäuser ihr Entlassmanagement evaluieren und darauf basierend modifizieren. Als Methoden kommen dabei schriftliche Befragungen, Telefonanrufe und Informationsbesuche am häufigsten von Patienten aber auch von Angehörigen, niedergelassenen Ärzten oder ambulanten Pflegediensten zum Einsatz (vgl. Abbildung 17).

Zusammenfassend lässt sich festhalten, dass vor allem die Verbreitung schriftlicher Standards und auch die flächendeckende Infrastruktur an spe- 


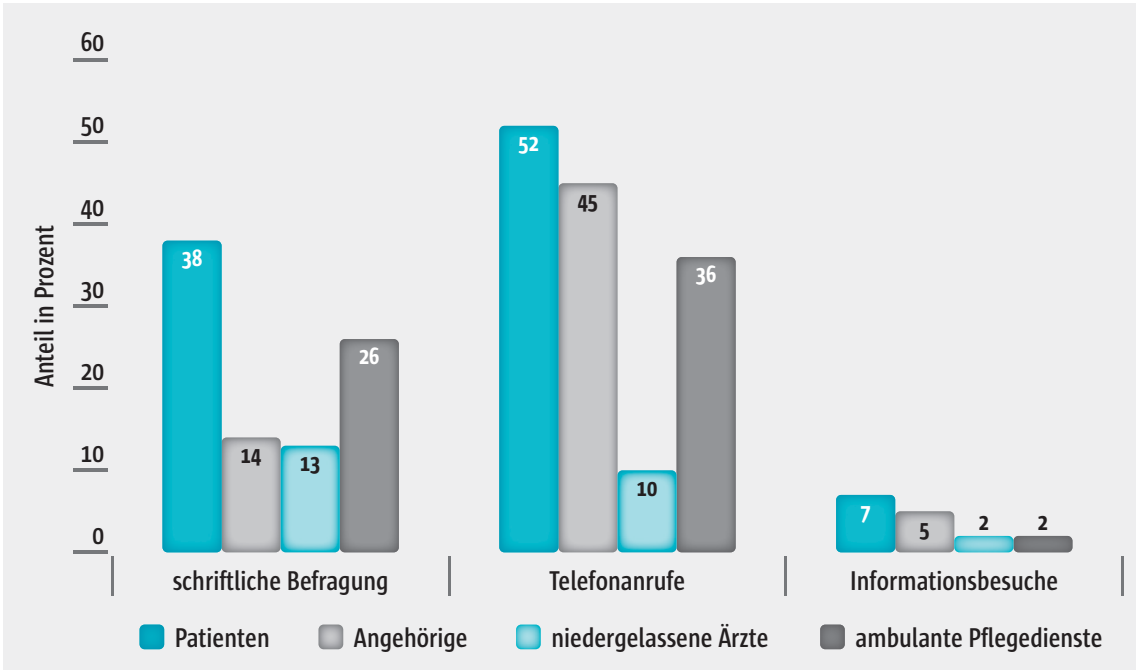

Abb. 17 Methodik der Evaluation des Entlassmanagements (eigene Darstellung in Anlehnung an DKI, 2014)

ziell zuständigen Organisationseinheiten und Stellen, sowie speziell dafür qualifizierten Fachkräften, zu den aktuellen Stärken des Entlassmanagements in Deutschland gehören. Auch die recht hohe Beteiligung an regionalen Initiativen, die Fallbesprechungen in multiprofessionellen Teams und der hohe Standardisierungsgrad in der Überleitung in stationäre Reha- und Pflegeeinrichtungen zum Beispiel werden als Stärken hervorgehoben (DKI, 2014). Dies spiegelt aktuelle Einschätzungen wieder, dass sich das Entlassmanagement in den letzten zehn Jahren bereits stark gewandelt und verbessert habe. Sei es damals noch oft ungeplant erfolgt, gibt es heute vielerorts feste Ansprechpartner für das Entlassmanagement.

Dennoch unterscheiden sich die Strukturen zwischen den Krankenhäusern zum Teil noch stark und nicht alle Krankenhäuser haben funktionierende Strukturen vorzuweisen (Osterloh, 2013). Defizite und Qualitätsunterschiede in der Organisation des Übergangs aus dem Krankenhaus bleiben mancherorts bestehen (Deimel et al., 2012). Verbesserungen scheinen derzeit insbesondere mit Blick auf die Kommunikation mit niedergelassenen Ärzten notwendig. Dort würde ein höherer Standardisierungsgrad hilfreich sein, erscheint es. Auch die Anschlussbehandlung mit Medikamenten muss nachgebessert werden, sowie die systematische Evaluation der bestehenden Herangehensweisen (DKI, 2014). 


\subsubsection{Die Rolle von Homecare beim Entlassmanagement}

Im Prozess des Entlassmanagements ist nicht nur von Relevanz, dass die Kommunikation und Informationsweitergabe zwischen unterschiedlichen Akteuren bewerkstelligt wird. Es geht auch um die Auswahl und Bereitstellung angemessener therapeutischer Maßnahmen, die den Übergang in den häuslichen (ambulanten) Bereich ermöglichen. Dies spielt vor allem bei Patienten mit besonderem post-stationärem Pflege- und Versorgungsbedarf eine kritische Rolle. Mit dem Entlassmanagement wird oftmals die Pflege und Versorgung vom Krankenhaus ins eigene Haus verlagert. Homecare kann hier als Substitut für die Krankenhausversorgung eingesetzt werden (Richards et al., 1998). Homecare-Unternehmen versorgen Patienten nicht nur mit medizinischen Produkten, sondern unterstützen diese auch in der Therapieanwendung. Oftmals sind es in der Praxis genau diese Akteure, die ein termingerechtes Entlassmanagement überhaupt ermöglichen (BVMed, 2014).

In der internationalen Literatur werden unter dem Stichwort „hospital at home“ Ansätze subsumiert, die verschiedene pflegerische wie rehabilitative Leistungen in der Häuslichkeit des Patienten vereinen, um damit entweder die Krankenhaus-Verweildauer zu verkürzen oder eine Wiedereinweisung zu vermeiden (Richards et al., 1998). Eine Studie zur Effektivität und Akzeptanz von „hospital at home“-Ansätzen zeigte vergleichbare Ergebnisse in Interventions- und Kontrollgruppe mit Blick auf Mortalität, funktionelle Fähigkeiten und Lebensqualität. Auch wenn die Homecare-Versorgung somit kein besseres Qualitätsniveau erreichen kann, ist sie doch zumindest gleichwertig zur stationären Versorgung und kann somit sowohl substitutiv als auch ergänzend dazu in geeigneten Fällen gleich effektiv sein. Darüber hinaus äußerten Homecare-Patienten eine signifikant höhere Zufriedenheit in Bezug auf die Einbindung in Entscheidungen (Richards et al., 1998).

Mit Blick auf die bereits erwähnte DKI-Studie ist bezeichnend, dass in Deutschland bei Patienten mit besonderem post-stationärem Pflege- oder Versorgungsbedarf ein standardisiertes Entlassmanagement mit niedergelassenen Ärzten nur bei circa 30\% der Krankenhäuser standardmäßig erfolgt (DKI, 2014). Weniger als die Hälfte der Krankenhäuser empfinden die Zusammenarbeit mit den niedergelassenen Ärzten als gut oder sehr gut. Insbesondere die Kontaktaufnahme und Informationsweitergabe sei dabei problematisch. Wesentlich besser gelingt im Vergleich dazu die Zusammenarbeit mit den verschiedenen Pflegeeinrichtungen (vgl. Abbildung 18).

Zwar kommt die DKI-Studie zu dem Schluss, dass nach Einschätzung der Krankenhäuser die Versorgung der Patienten mit Hilfsmitteln im unmittelbaren Anschluss an die Behandlung im Krankenhaus selten ein Problem darstellt. Doch sind es immer noch zwischen 24 und 47\% aller Krankenhäuser, die manchmal bis häufig von verschiedenen Problemen in der Hilfsmittelver- 


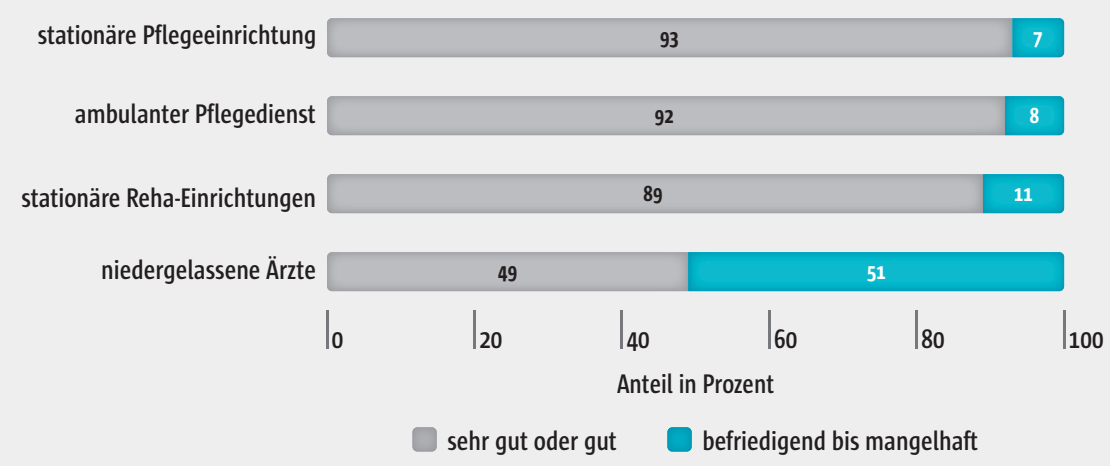

Abb. 18 Beurteilung der Zusammenarbeit mit Kooperationspartnern im Entlassmanagement (eigene Darstellung in Anlehnung an DKI, 2014)

sorgung betroffen sind (vgl. Abbildung 19). Die meisten Probleme entstehen dabei bei Entlassungen an Feiertagen und Wochenenden.

Noch problematischer stellt sich die Versorgung mit Medikamenten dar, was primär auf eine fehlende Finanzierung der Medikamentenmitgabe bei Entlassung und die eingeschränkten Verordnungsmöglichkeiten zurückzuführen sei. Welchen Einfluss die Änderungen, die mit dem GKV-VSG in Kraft treten, haben werden, bleibt abzuwarten. Kostenzusagen und Pflegeeinstufungen durch die Krankenkassen erfolgen zu einem Großteil zwar innerhalb einer Woche, doch dauert es bei 33-46\% der Krankenhäuser auch standardmäßig länger als zwei Wochen, was für den Patienten, der mit unmittelbarem Pflegebedarf entlassen wird, eine lange Zeitspanne sein kann. Auch die fachärztliche Weiterversorgung ist durch oftmals sehr lange Wartezeiten häufig nur mit Verzögerung umsetzbar. Vor allem aber Patienten mit Homecare-Be-

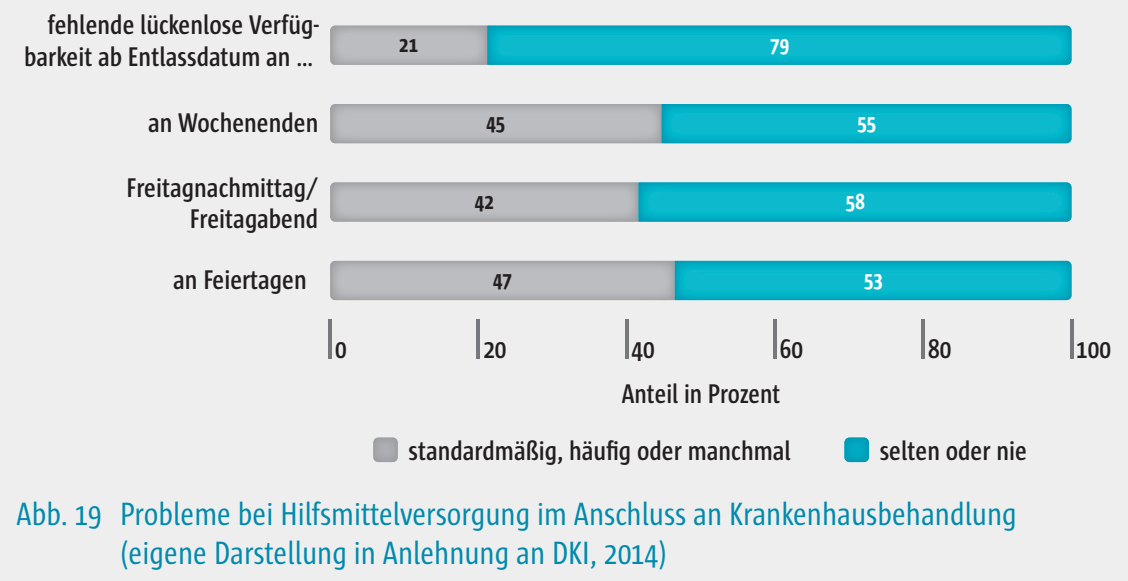


darf benötigen häufig die Einbindung eines Diabetologen, Dermatologen, Urologen oder anderer Fachrichtungen.

Trotz der positiven Entwicklungen bei der organisatorischen Umsetzung des Entlassmanagements aufseiten der Krankenhäuser, ist demnach die qualitativ gleichwertige Weiterversorgung von Patienten nach der Entlassung derzeit noch mangelhaft und verbesserungsbedürftig. Homecare-Unternehmen können an dieser Stelle helfen, die Defizite insbesondere im Hinblick auf die Weiterversorgung von Patienten mit besonderem Pflege- und Versorgungsbedarf zu beseitigen. So hebt der BVMed in seiner Stellungnahme zum Referentenentwurf des GKV-VSG von November 2014 die besondere Rolle von Homecare-Unternehmen in diesem Zusammenhang hervor und fordert, dass diese als Leistungserbringer in den Rahmenvertrag, der die Zuständigkeiten für das Entlassmanagement regeln soll, eingebunden werden (BVMed, 2014). 